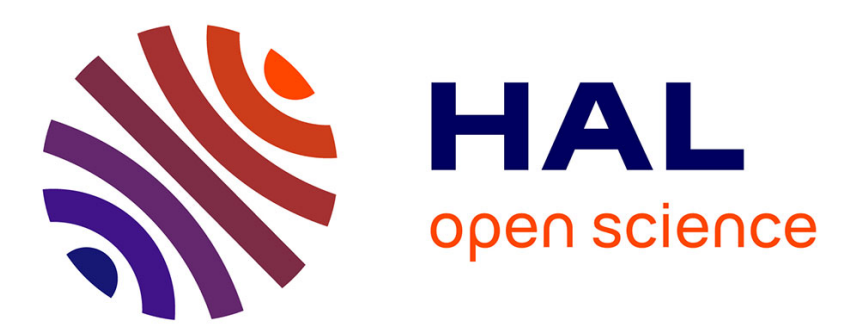

\title{
On the Validation of A Priori Estimates of Standard Displacement Uncertainties in T3-Stereocorrelation
}

C Minguet, F. Soulas, T Lafargue-Tallet, E Chalumeau, M Pommies, R Peiffer, François Hild

\section{- To cite this version:}

C Minguet, F. Soulas, T Lafargue-Tallet, E Chalumeau, M Pommies, et al.. On the Validation of A Priori Estimates of Standard Displacement Uncertainties in T3-Stereocorrelation. Measurement Science and Technology, 2021, 32 (2), pp.024004. 10.1088/1361-6501/abafe1 . hal-02916960

\section{HAL Id: hal-02916960 https://hal.science/hal-02916960}

Submitted on 18 Aug 2020

HAL is a multi-disciplinary open access archive for the deposit and dissemination of scientific research documents, whether they are published or not. The documents may come from teaching and research institutions in France or abroad, or from public or private research centers.
L'archive ouverte pluridisciplinaire HAL, est destinée au dépôt et à la diffusion de documents scientifiques de niveau recherche, publiés ou non, émanant des établissements d'enseignement et de recherche français ou étrangers, des laboratoires publics ou privés. 


\title{
On the Validation of A Priori Estimates of Standard Displacement Uncertainties in T3-Stereocorrelation
}

\author{
C. Minguet, ${ }^{1}$ F. Soulas, ${ }^{1}$ T. Lafargue-Tallet,${ }^{2-3}$ E. Chalumeau, ${ }^{2}$ \\ M. Pommies, ${ }^{2}$ R. Peiffer ${ }^{3}$ \& F. Hild ${ }^{4}$ \\ ${ }^{1}$ EikoSim, Versailles, France \\ ${ }^{2}$ ALPhANOV, Institut d'Optique d'Aquitaine, Talence, France \\ ${ }^{3}$ MBDA France, Le Plessis-Robinson, France \\ ${ }^{4}$ Université Paris-Saclay, ENS Paris-Saclay, CNRS \\ LMT - Laboratoire de Mécanique et Technologie, Gif-sur-Yvette, France \\ E-mail: francois.hild@ens-paris-saclay.fr
}

July 2020

\begin{abstract}
Uncertainty quantification is crucial for any measurement technique. The present work aims at validating a priori estimates of displacement uncertainties. Images acquired prior to fourteen thermomechanical tests were analyzed via FE-based stereocorrelation to determine actual displacement uncertainties, which were compared to a priori estimates. For the studied experimental database, a very good agreement was observed between a priori and a posteriori estimates of standard displacement uncertainties.
\end{abstract}

Keywords: Covariance matrix, Displacement, Uncertainty quantification 


\section{Introduction}

One key aspect of metrology, i.e., the science of measurement, is to report the quality of mesurands so that the user is informed about their reliability. Without such information, measurement results are useless [1]. It is therefore very important to assess (and report) measurement uncertainties (i.e., "parameter characterizing the dispersion of the quantity values being attributed to a measurand" [2]). There are different types of uncertainty, namely, aleatoric (or random) and systemic (e.g., poorly designed experiments or inherent shortcomings in a measurement system) uncertainties. The random uncertainty is inherent to the physical phenomena involved in the measurement technique (e.g., acquisition noise of cameras) and cannot be reduced. The epistemic uncertainty, which results, for instance, from incomplete knowledge, may be reduced. In the following, random uncertainties are analyzed for displacement measurements via stereocorrelation [3-5].

Most stereocorrelation codes are subset-based to perform spatial and temporal registrations to measure $3 \mathrm{D}$ shapes and their subsequent deformations. Clouds of 3D points (i.e., centers of interrogation windows) and displacement vectors are available [69]. Recently, global frameworks have been introduced [10]. They are based on the mathematical model of the surface of interest (e.g., NURBS patches used in CAD modelers $[11,12]$ or finite element (FE) discretizations [13-15]). Surface meshes made of three-noded elements are the simplest implementation. Their deformation described by the corresponding FE kinematic bases are a natural (and easy) extension. They are considered herein.

There are two main routes to evaluate random uncertainties. The first is via theoretical or numerical tools. They consist in propagating acquisition noise for evaluating uncertainties associated with the calibration of stereo-systems and subsequent shape measurements [16-19]. Such approaches allow the systemic errors to be avoided. 
However, they may occur in practice, and the previous analyses call for experimental validations $[20,21]$. As maturity increases in the use of stereocorrelation, some systemic errors (e.g., due to lighting or the deformation of the surface of interest [22]) may be reduced. Guidelines were then formulated to mitigate as much as possible the level of (systemic) uncertainties in comparison to so-called noise-floor levels [23].

If acquisition noise can be modeled with Gaussian variables, then one important piece of information is related to the covariance matrix of the measured quantities when analyzing the performance of DIC and stereoDIC tools [24]. For FE-based stereocorrelation, it was shown that the covariance matrix of the measured nodal displacements was equal to the inverse of the Hessian matrix used in the minimization scheme of the weighted cost function in which the gray level differences were scaled by their corresponding variances $[21,25]$. Such result corresponds to the so-called Rao and Cramér lower bounds [26,27] applied to the evaluation of displacement uncertainties. From these derivations, very simple expressions were derived to have first order estimates based upon mean field approximations. They will be utilized hereafter and extended to account for the fact that when the number of integration points per element becomes too high, a pixel limit will be reached and the measurement uncertainty can no longer be lowered.

The present paper aims at validating a priori uncertainty estimates for experiments in which plates were heated by laser shocks. In the first part, the derivation of the standard displacement uncertainty is recalled, and the main influencing parameters are discussed. Fourteen laser shocks were performed on five different stainless steel plates onto which different patterns were deposited. They are described in the second part. The third part is devoted to the uncertainty quantification. In particular, a priori estimates are compared with a posteriori assessments. 


\section{Displacement Uncertainties}

The displacement uncertainties analyzed herein correspond to the standard deviations of nodal displacements measured within a FE-stereocorrelation framework. The latter consists in minimizing the weighted cost function $\phi^{2}$

$$
\phi^{2}=\sum_{c=1}^{n_{c}} \sum_{\boldsymbol{x}^{c} \in \mathcal{S}^{c}} \frac{\left(I_{t}^{c}\left(\boldsymbol{x}^{c}+\boldsymbol{u}^{c}\left(\boldsymbol{x}^{c},\{\boldsymbol{U}\}\right)\right)-I_{0}^{c}\left(\boldsymbol{x}^{c}\right)\right)^{2}}{2\left(\sigma^{c}\right)^{2}}
$$

with respect to the unknown degrees of freedom gathered in the column vector $\{\boldsymbol{U}\}$, where $I_{t}^{c}$ denote images in the deformed configuration, and $I_{0}^{c}$ those in the reference configuration acquired by $n_{c}$ cameras (e.g., $n_{c}=2$ in stereocorrelation). The variance $\left(\sigma^{c}\right)^{2}$ is proportional to the variance of acquisition noise and depends upon the gray level interpolation scheme [25].

In the present case, the 3D displacement field $\boldsymbol{U}$ is parameterized as

$$
\boldsymbol{U}(\boldsymbol{X})=\sum_{i} \boldsymbol{U}_{i} N_{i}(\boldsymbol{X})
$$

where $\boldsymbol{X}$ denotes the vector defining the position of any point on the 3D surface of interest, $\boldsymbol{U}_{i}$ the 3D displacement of the $i$-th node, and $N_{i}(\boldsymbol{X})$ the corresponding shape function. The position of any point on the physical surface is projected onto the camera plane thanks to the projection matrix $\left[\boldsymbol{P}^{c}\right]$ that relates the homogeneous coordinates in the camera plane $\left\{\boldsymbol{x}^{c}\right\}$ to those in the physical space $\{\boldsymbol{X}\}[3,28,29]$

$$
s^{c}\left\{\boldsymbol{x}^{c}\right\}=\left[\boldsymbol{P}^{c}\right]\{\boldsymbol{X}\}
$$

where $s^{c}$ is the local scale factor. Consequently, the displacement field $\boldsymbol{U}$ induces apparent motions $\left.\boldsymbol{u}^{c}(\{\boldsymbol{U}\})\right)$ in each camera plane.

The evaluation of the covariance matrix consists in propagating acquisition noise in the pictures $I^{c}(\boldsymbol{x})$ to the measured nodal displacements in a perturbation analysis on the measured degrees of freedom $U_{k}$ [25]. This propagation is the inverse problem that consists in computing the effect of small perturbations on the $k$-th degree of freedom 
$U_{k}$ along direction $\boldsymbol{e}_{k}$ on gray level variations. The first step is to derive the so-called kinematic sensitivities (no index summation)

$$
\boldsymbol{\varphi}_{k}^{c}\left(\boldsymbol{x}^{c}\right) \equiv \frac{\delta \boldsymbol{u}^{c}}{\delta U_{k}}\left(\boldsymbol{x}^{c}\right)=\left[\boldsymbol{\Pi}^{c}(\boldsymbol{X})\right] \boldsymbol{e}_{k} N_{k}(\boldsymbol{X})
$$

where $\left[\boldsymbol{\Pi}^{c}\right]=\partial \boldsymbol{x}^{c} / \partial \boldsymbol{X}$ is the placement sensitivity matrix that expresses how a small placement shift $\delta \boldsymbol{X}$ induces apparent shifts $\delta \boldsymbol{x}^{c}$ in each camera plane. These elementary motions then induce gray level variations

$$
\delta I^{c}\left(\boldsymbol{x}^{c}\right)=\nabla I_{0}^{c}\left(\boldsymbol{x}^{c}\right) \cdot \delta \boldsymbol{u}^{c}\left(\boldsymbol{x}^{c}\right)
$$

that are recast as gray level sensitivities

$$
\frac{\delta I^{c}}{\delta U_{k}}\left(\boldsymbol{x}^{c}\right)=\nabla I_{0}^{c}\left(\boldsymbol{x}^{c}\right) \cdot \boldsymbol{\varphi}_{k}^{c}\left(\boldsymbol{x}^{c}\right)
$$

White Gaussian noise is assumed at each pixel position. It is worth noting that if Poisson noise occurs, a simple Anscombe transform [30] will allow the noise variance to be made uniform $[31,32]$. Within this setting, it was shown that the covariance matrix of the measured degrees of freedom $[\boldsymbol{C}]=\left\langle\{\delta \boldsymbol{U}\}\{\delta \boldsymbol{U}\}^{\top}\right\rangle_{\eta}$ (where $\langle\cdot\rangle_{\eta}$ denotes the expected value over noise $\eta$ ) reduced to [25]

$$
[\boldsymbol{C}]=[\boldsymbol{H}]^{-1}
$$

where $[\boldsymbol{H}]$ is the total Hessian matrix of the stereocorrelation scheme

$$
H_{i j}=\sum_{c=1}^{n_{c}} \sum_{\boldsymbol{x}^{c} \in \mathcal{S}^{c}} \frac{\left(\boldsymbol{\nabla} I_{0}^{c}\left(\boldsymbol{x}^{c}\right) \cdot \boldsymbol{\varphi}_{i}^{c}\left(\boldsymbol{x}^{c}\right)\right)\left(\boldsymbol{\nabla} I_{0}^{c}\left(\boldsymbol{x}^{c}\right) \cdot \boldsymbol{\varphi}_{j}^{c}\left(\boldsymbol{x}^{c}\right)\right)}{2\left(\sigma^{c}\right)^{2}}
$$

Equation (7) corresponds to the so-called Rao and Cramér lower bounds [26, 27] applied to the evaluation of displacement uncertainties. From this very general expression, first order solutions can be derived by performing mean-field approximations. Long-range (i.e., $\left.\left[\boldsymbol{\Pi}^{c}(\boldsymbol{X})\right] \boldsymbol{e}_{k}\right)$ and short range (i.e., $\left.\boldsymbol{\nabla} I_{0}^{c}\right)$ fluctuations are separated from those of the shape functions $N_{k}$. Consequently, the integral of the tensor $\nabla I_{0}^{c} \otimes \nabla I_{0}^{c}$ 
is approximated by $S_{k}^{c} / 2\left\langle\left\|\nabla I_{0}^{c}\right\|_{2}^{2}\right\rangle \boldsymbol{I}$, where $\boldsymbol{I}$ is the identity tensor, and $S_{k}^{c}$ is the area of the projected support surface of the shape function $N_{k}$. With such hypotheses, a first order estimate of the variance $\sigma_{k}^{2}$ of the nodal displacement $U_{k}$ uncertainty was proposed [25]

$$
\sigma_{k}^{2}=\frac{\Sigma_{u}^{2}}{N_{I P}^{2}} \sum_{c} \frac{4\left(\sigma^{c}\right)^{2}}{\left(\Pi_{k}^{c}\right)^{2}\left\langle\left\|\nabla I_{0}^{c}\right\|_{2}^{2}\right\rangle}
$$

where $\Pi_{k}^{c}$ denotes the mean (pixel $/ \mathrm{m}$ ) placement sensitivity for the studied component of displacement, and $N_{I P}$ the equivalent number of integration points (i.e., the square root of the total number of integration points per element). For artificial cases, the multiplicative constant $\Sigma_{u}$ was found equal to $1.2 \pm 0.3$ for global T3stereocorrelation [25]. More refined analyses have shown that a lower bound to $\Sigma_{u}$ was 1.08 [21] when three-noded elements were used. This last value was selected for the results reported herein.

It is worth noting that in global stereocorrelation, the integrations to compute, for instance the total Hessian matrix (8), are not performed pixel-wise but at integration points of each T3 element. Consequently, the number of integration points will influence the variance level (Equation (9)), and the standard deviation is inversely proportional to the equivalent number $N_{I P}$. Given the fact that integration points do not coincide with pixel positions, a gray level interpolation scheme is required to compute $I^{c}\left(\boldsymbol{x}^{c}\right)$. Consequently, the variance $\left(\sigma^{c}\right)^{2}$ has to be computed by accounting for this feature [25].

To illustrate the predictions of Equation (9), Figure 1 shows the standard displacement uncertainty of a stereosystem representative of the experimental facility of the present study (i.e., a plate to be laser shocked) as a function of the square root of the number of integration points. The standard deviation $\sqrt{2} \sigma^{c}=0.8$ gray level, and $\sqrt{\left\langle\left\|\nabla I_{0}^{c}\right\|_{2}^{2}\right\rangle}=11.5$ gray level/px. For the two in-plane directions (i.e., $X$ and $Y$ ) the measurement uncertainties are about 2.8 times lower than that for the out-of-plane (i.e., $Z$ ) direction. Such differences are mostly due to the placement sensitivities $\partial \boldsymbol{x}^{c} / \partial X$, $\partial \boldsymbol{x}^{c} / \partial Y$, that are higher than $\partial \boldsymbol{x}^{c} / \partial Z$. The typical unit for placement sensitivities is millipixel per micrometer (or $\mathrm{mpx} / \mu \mathrm{m}$ ). In the present case, the RMS placement 
sensitivities $\sqrt{\left\langle\|\partial \boldsymbol{x} / \partial X\|^{2}\right\rangle}=8.1 \mathrm{mpx} / \mu \mathrm{m}, \sqrt{\left\langle\|\partial \boldsymbol{x} / \partial Y\|^{2}\right\rangle}=8.7 \mathrm{mpx} / \mathrm{um}$ are about 2.6 times higher than $\sqrt{\left\langle\|\partial \boldsymbol{x} / \partial Z\|^{2}\right\rangle}=3.2 \mathrm{mpx} / \mathrm{um}$ for both cameras.

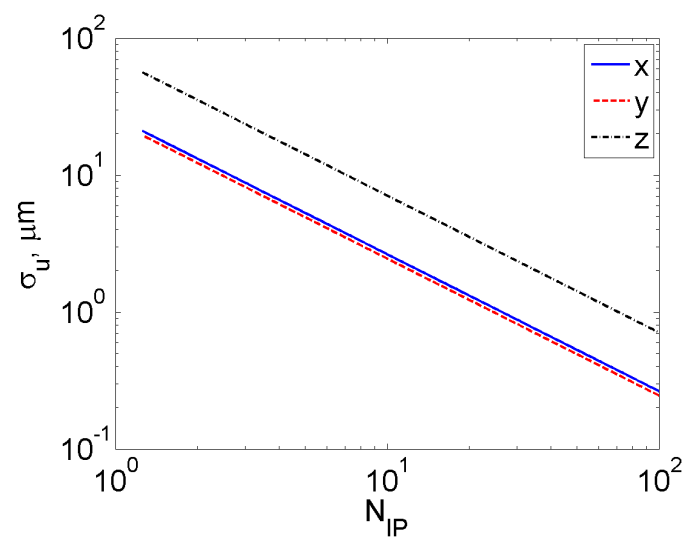

Figure 1. Standard displacement uncertainties $\sigma_{u}$ vs. equivalent number of integration points $N_{I P}$ for the three components of displacements according to Equation (9)

\section{Analyzed Experiments}

\subsection{Experimental Facility}

The experimental setup was installed in a laser-proof cabin. The laser scanner head was mounted on a 6-axis robot for a quick and easy positioning in space. It was located at a distance of $1170 \mathrm{~mm}$ from the plate to obtain a spot size of $40 \mathrm{~mm}$ on the target (Figure 2(a)). The head had an inclination of $5^{\circ}$ downward to avoid a direct return of reflections on its optics. An air flow was created by a blowing hood above the sample to mitigate heat haze effects [33-36]. 


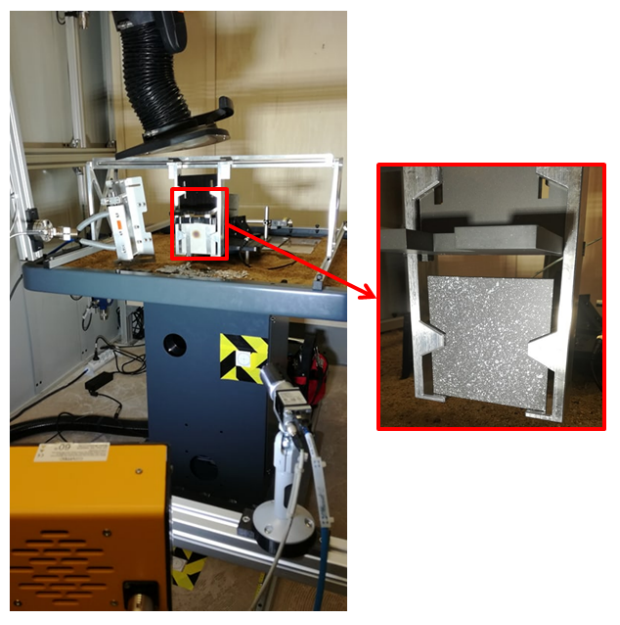

(a)

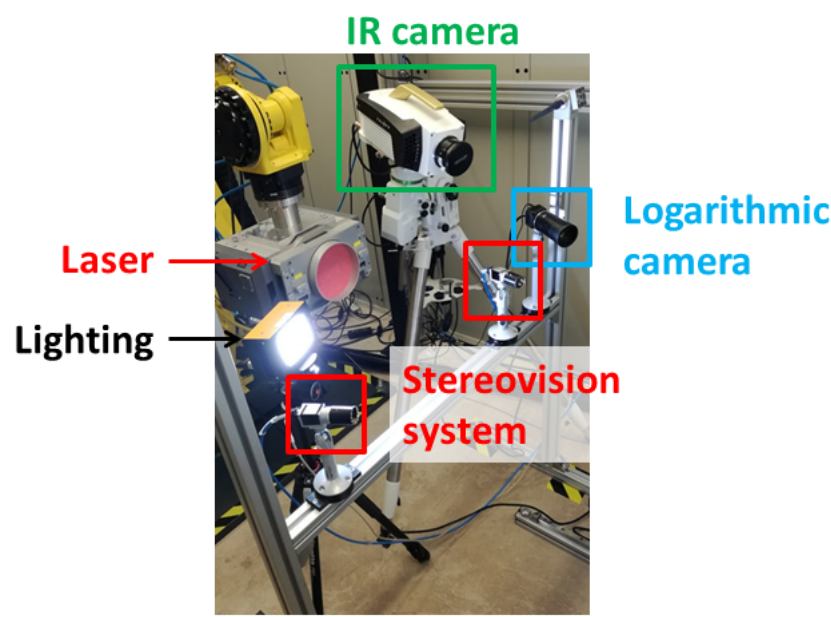

(b)

Figure 2. (a) Support of the plate to be illuminated by the laser. (b) Laser head and multiview system monitoring the experiment

\subsection{Optical setup}

A multiview system was used to monitor the experiments reported herein (Figure 2(b)). Three visible light cameras and one multispectral IR camera were installed. The IR camera was used for thermographic measurements (not discussed herein). One MAGIC camera (MC1003-1VF) was used to focus on the illuminated area thanks to its wide dynamic range (logarithmic response [37]). The measurements reported herein were performed with the acquisitions of the two Basler cameras (Table 1). Blue filters were mounted on the objective lenses of both cameras to mitigate gray level variations induced by near IR radiations. 
Table 1. DIC hardware parameters

\begin{tabular}{|l|l|}
\hline \hline Cameras & Basler acA1300-60gc \\
Definition & $1024 \times 1280$ pixels (color images) \\
Color filter & High-pass filter $(1095 \mathrm{~nm})$ \\
Gray Levels rendering & $3 \times 8$ bits \\
Lens & $25 \mathrm{~mm} \mathrm{f} / 1.4$ \\
Aperture & $\mathrm{f} / 8$ \\
Field of view & $174 \times 218 \mathrm{~mm}^{2}$ \\
Image scale & $\approx 170 \mu \mathrm{m} /$ pixel \\
Stereo-angle & $38^{\circ}$ \\
Stand-off distance & $710 \mathrm{~mm}$ \\
Image acquisition rate & $40 \mathrm{fps}$ \\
Patterning technique & see text and Figure 6 \\
Pattern feature sizes & see Figure 8 \\
\hline \hline
\end{tabular}

The studied samples were $100 \times 100 \times 2$-mm plates made of $304 \mathrm{~L}$ stainless steel. They were mirror polished to enable for nanotexturation. A specially designed calibration target was used to determine the projection matrices $\left[\boldsymbol{P}^{c}\right]$ (Figure 3(a)). It was made of 6061-T6 aluminum alloy. With such design, the plate to be tested can also be part of the calibration procedure. In the present case, a global approach was followed to determine the projection matrices of both cameras $[14,38]$. Given the geometry of the calibration target (Figure 3(a)), only one pair of pictures was utilized to perform such step. As the calibration target and the plate to be tested were imaged, the projection matrices are expressed in the frame of the numerical model consisting of the calibration target and the plate [10]. This features allows for direct comparisons between experimental measurements and numerical simulations for the initial shape and its deformation in experiments. Fourteen fiducials were used to initialize the minimization scheme (ten of them on the calibration target, and four of them on the plate (Figure $3(\mathrm{~b}-\mathrm{c})$ ). At the end of the calibration step, these points were drawn on the images of the reference 
configuration for qualitative check (Figure 3(b-c)). Once the calibration was completed, the calibration target was not removed from the scene. However, for each new test, the calibration step was repeated since permanent deformations of the plates were observed.

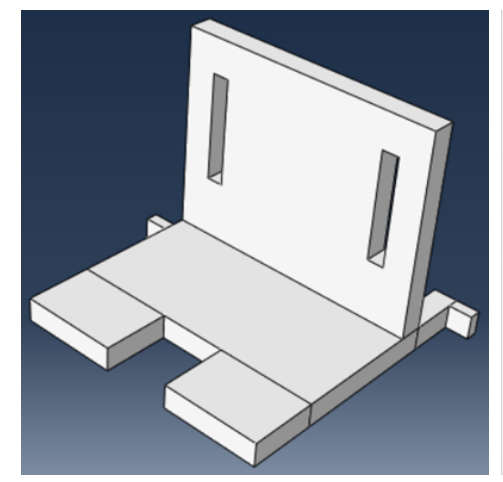

(a)

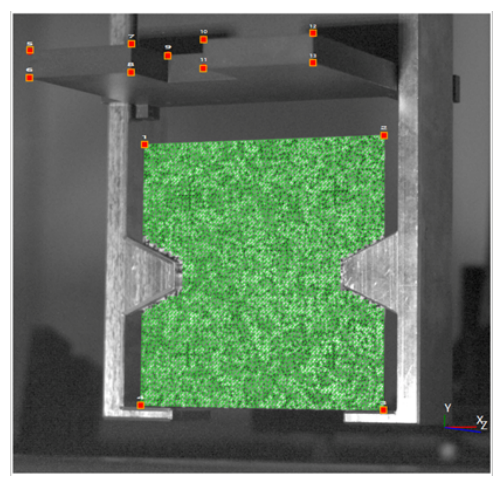

(b)

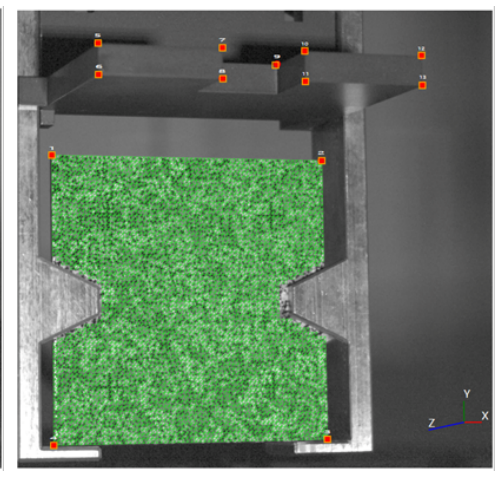

(c)

Figure 3. (a) CAD model of the calibration target used herein. (b-c) Fiducials drawn on the images of the reference configuration of the lower part of the calibration target and the plate to be tested at the end of the calibration procedure for both cameras. The reference mesh is also shown on one of the $100 \times 100$-mm plates

\subsection{Placement Sensitivity Fields}

Once the stereosystem was calibrated, the projection matrices $\left[\boldsymbol{P}^{c}\right]$ are known, and any component of the placement sensitivity matrices $\left[\boldsymbol{\Pi}^{c}(\boldsymbol{X})\right]$ can be computed at any location of the region of interest. Figure 4 shows the placement sensitivity fields (i.e., norms of $\partial \boldsymbol{x}^{c} / \partial X, \partial \boldsymbol{x}^{c} / \partial Y$, and $\partial \boldsymbol{x}^{c} / \partial Z$ ) for the two cameras, and the global (i.e., RMS) sensitivities drawn on the mesh of the nominal surface. These fields, as assumed in the mean field approximation, do not vary much over the region of interest, and are, on average, about 2.7 times higher in the in-plane directions in comparison to those in the out-of-plane directions. This information is very useful, for instance, when designing experiments and having to place cameras in an optimal way (i.e., minimizing the measurement uncertainties [39]). 


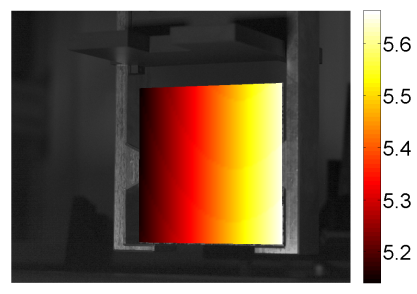

(a)

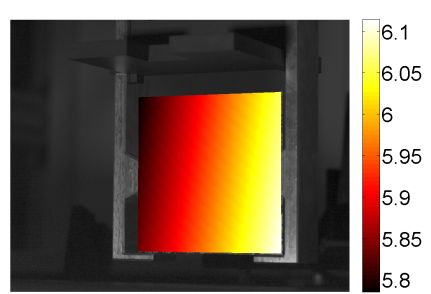

(d)

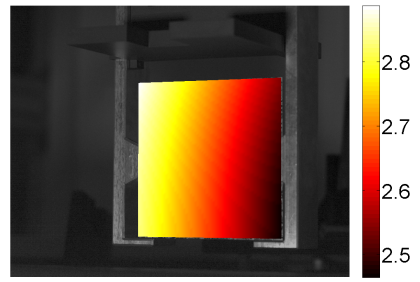

(g)

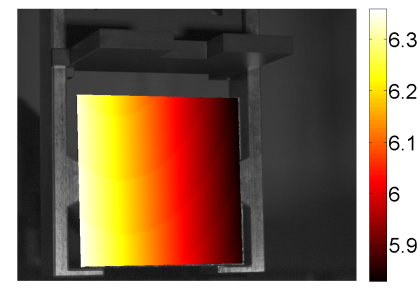

(b)

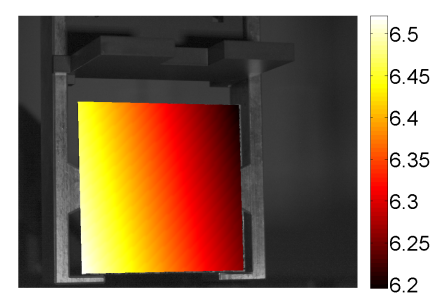

(e)

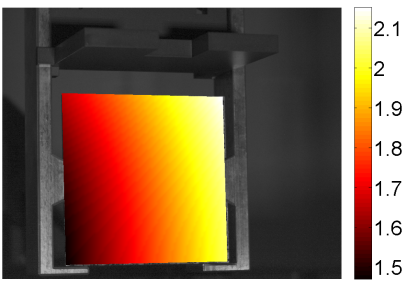

(h)

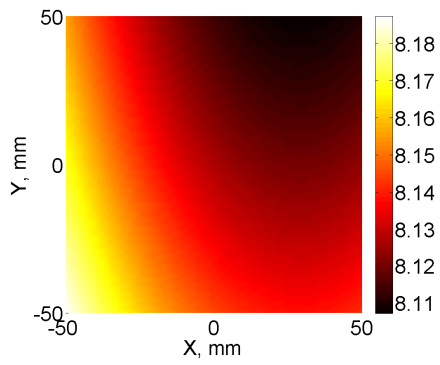

(c)

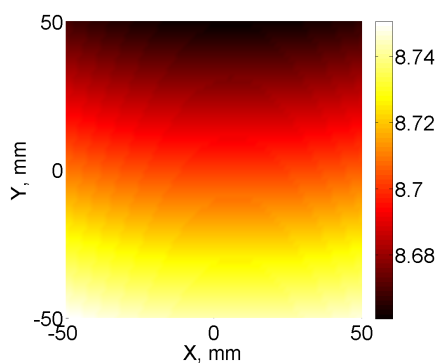

(f)

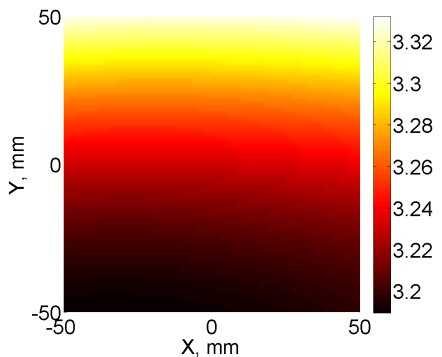

(i)

Figure 4. Placement sensitivity fields expressed in $\mathrm{mpx} / \mu \mathrm{m}$ of the first series of tests. Each row reports the results for $X, Y$ and $Z$ directions. The first column corresponds to camera \#1, the second to camera \#2, and the third to the total sensitivity projected onto the nominal shape of the plate

\subsection{Speckle Patterns}

Three plates were prepared with nanostructured patterns [40-42] (one of them with a central black zone for IR measurements) using predefined stencils (Figure 5), and the last two were obtained with regular B/W paints. 


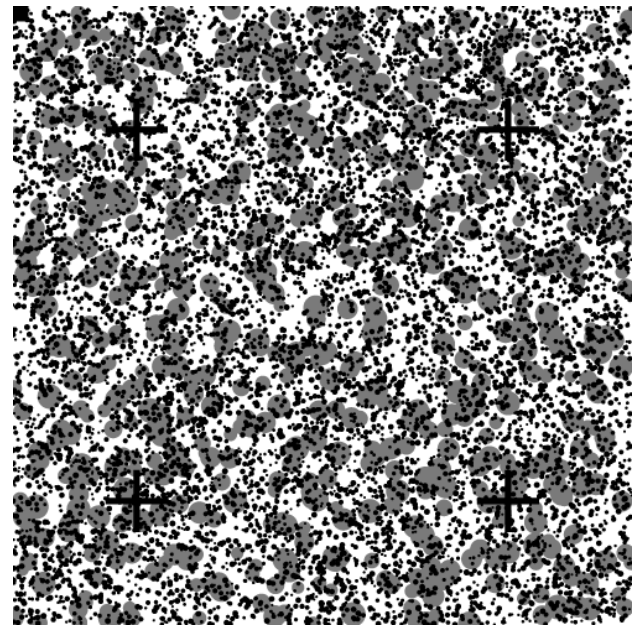

(a)

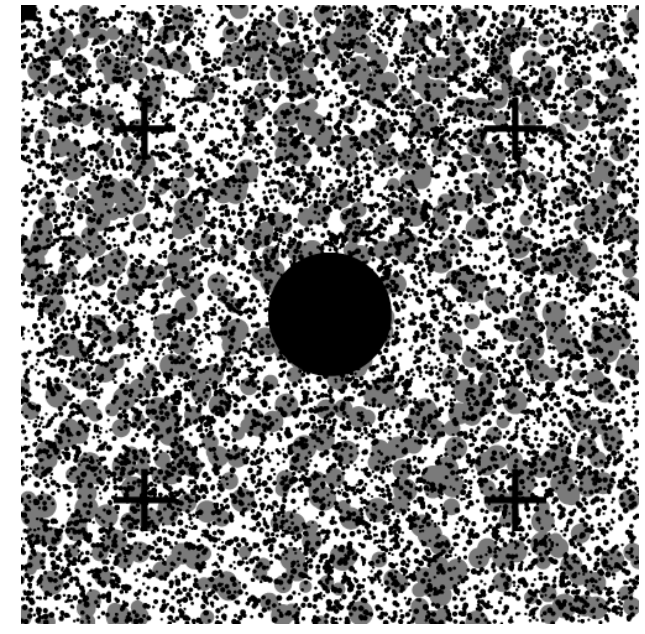

(b)

Figure 5. Two stencils used for the nanotextured patterns to be printed on $100 \times 100$ mm plates

Figure 6 shows the five patterns as acquired by the two cameras before the beginning of the test series. The corresponding gray level histograms are also reported where the bimodal feature is observed for the nanostructured patterns. Conversely, the histograms of painted patterns did not exhibit such property. Because high temperatures were reached during the experiments reported herein, filters were added to protect the Basler cameras (Table 1). Such protections led to histograms that did not span over the full dynamic range of the cameras. 

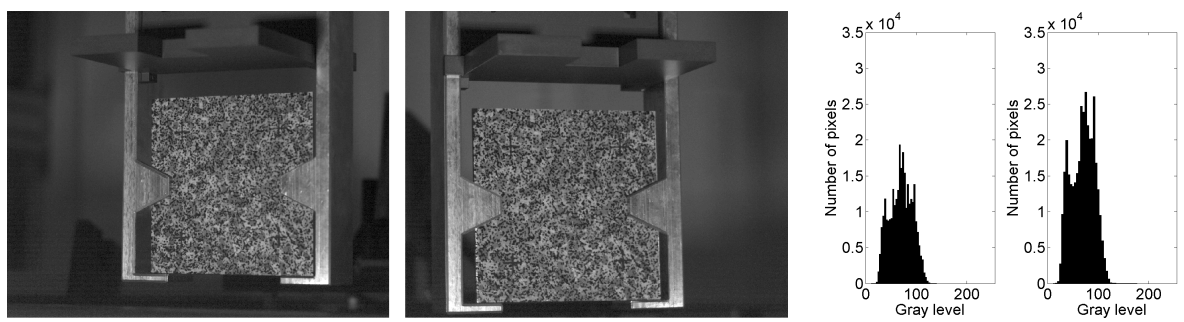

Pattern \#1
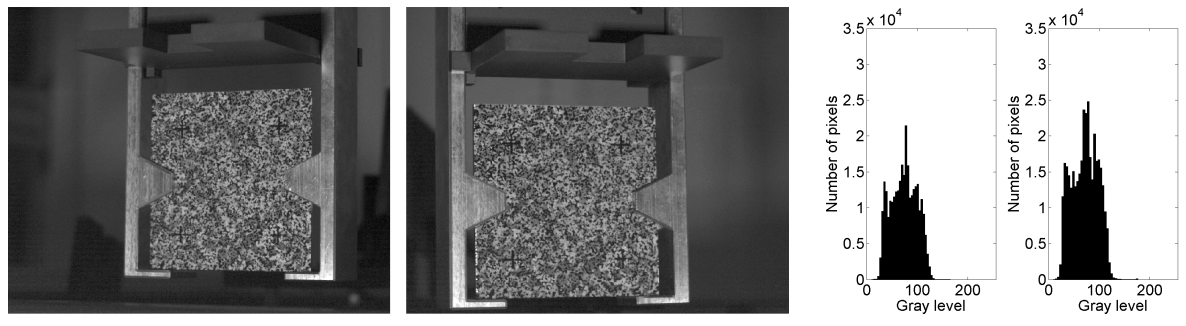

Pattern \#2
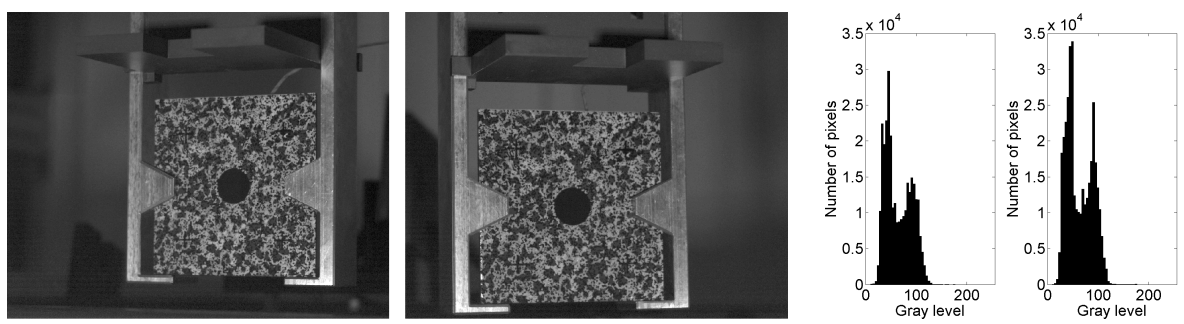

Pattern \#3
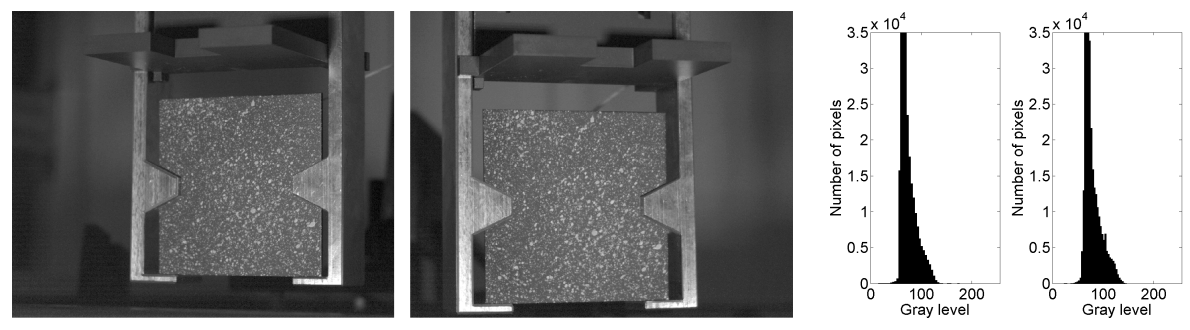

Pattern \#4
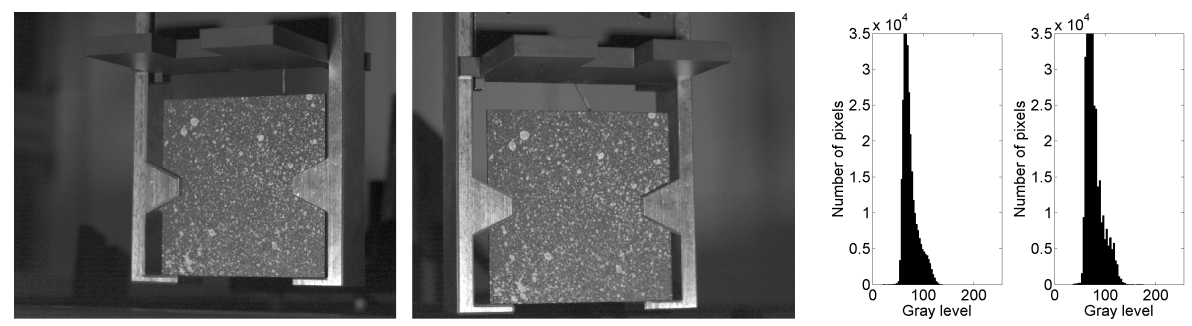

Pattern \#5

Figure 6. Reference images of the five patterns on $100 \times 100-\mathrm{mm}$ plates

To compute the characteristic feature size (or pattern size [23], see Table 1), the 
normalized autocorrelation was computed for each region of interest (ROI) [43]. In the present case, a sub-ROI whose size was a power of two was considered because fast Fourier transforms were utilized to compute the autocorrelation. Figure 7(a) shows the autocorrelation of the ROI of camera \#1 for the first test with a well-defined peak about the origin (Figure $7(\mathrm{~b})$ ). The characteristic feature size was then determined as the mean full width at half maximum (FWHM) along the two perpendicular directions (Figure $7(\mathrm{c}))$.

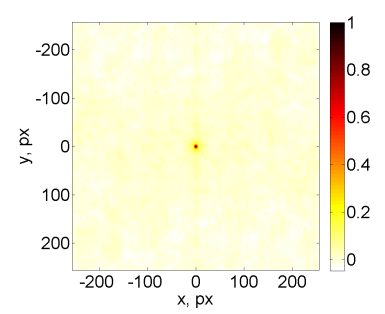

(a)

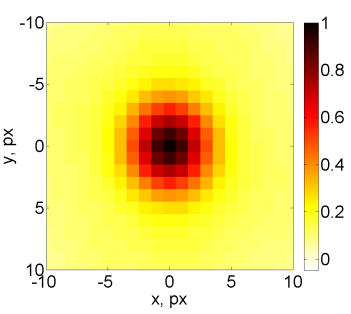

(b)

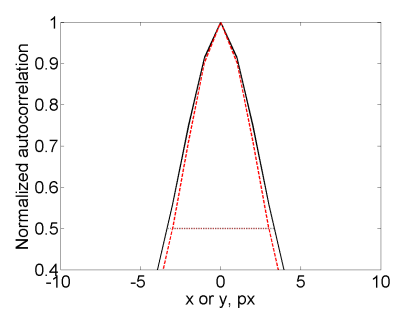

(c)

Figure 7. (a) Normalized autocorrelation of the region of interest of camera \#1 of the first pattern. (b) Zoom about the correlation peak. (c) Full width at half maximum for the two directions

The characteristic speckle sizes of all studied patterns and both cameras are reported in Figure 8. Apart from the third pattern, which had a larger level (i.e., $9 \mathrm{px}$ ), all other ones had a characteristic size of the order of $6 \mathrm{px}$ irrespective of the camera and the patterning technique.

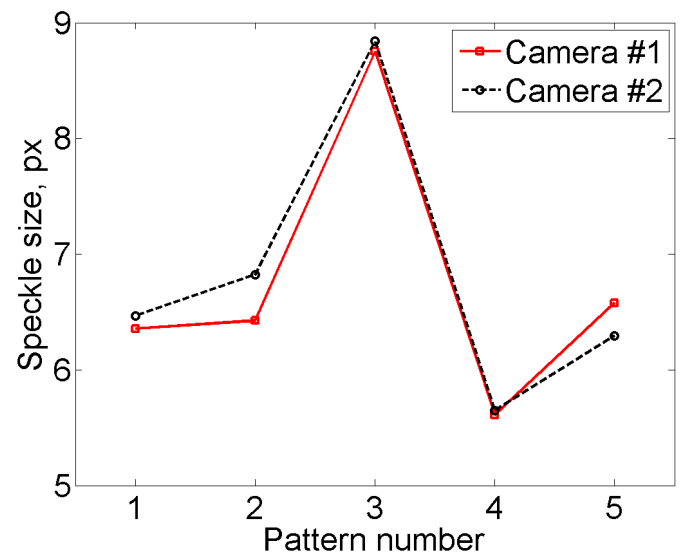

Figure 8. Characteristic speckle sizes for the five different patterns 
Another way of characterizing the patterns is through their contrast (i.e., $\nabla I_{0}^{c}$ ) that appears in the gray level sensitivities (Equation (6)) and more precisely, RMS image gradients $\sqrt{\left\langle\left\|\nabla I_{0}^{c}\right\|_{2}^{2}\right\rangle}$ in Equation (9). These quantities were evaluated for the five patterns and both cameras over the ROIs considered in the stereocorrelation measurements. Figure 9 shows that the RMS image gradients of any pattern were close for both cameras. The three nanostructured patterns led to slightly higher RMS levels in comparison to paint speckled patterns. The order of magnitude was about 10 gray level/px. This is an intermediate level for 8-bit images (e.g., about 20 gray level/px were observed for paint speckled pattern, and about 5 gray level/px for the Descent of the Cross painting [25]). This observation shows that the measurement uncertainties may be significantly lowered (i.e., by a factor of 2 ) if the dynamic range of the pictures were to span the whole 8 bits.

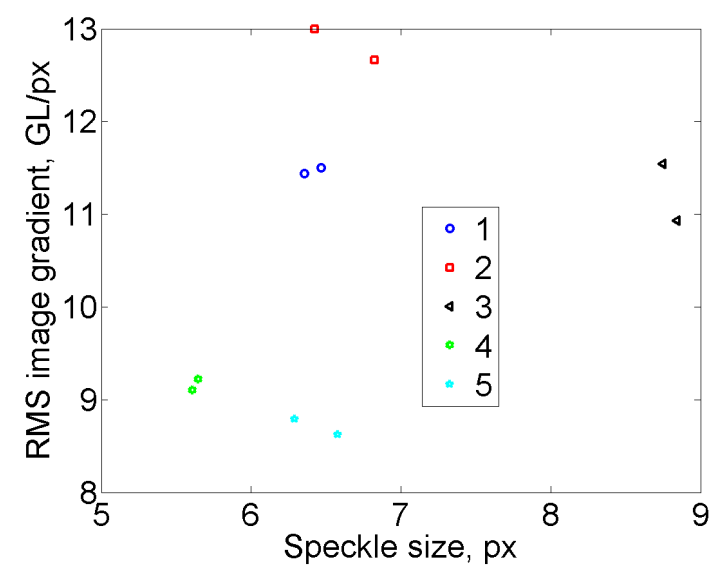

Figure 9. RMS image gradient vs. characteristic speckle sizes for the five different patterns and both cameras

\section{Uncertainty Quantifications}

\subsection{Experimental Protocol}

Table 2 gathers all the information concerning the 14 tests that were conducted. The laser spot size was always set to $40 \mathrm{~mm}$, the $(1 \mathrm{~kW})$ power distribution was Gaussian. Different durations were applied so that various temperature levels were achieved for the five series of tests. The temperatures, which are indicative, were measured by a bichromatic pyrometer monitoring the center of the laser spot. For each series, laser 
shocks were repeated (between 2 and 4 times) with different dwell durations. Smoke was observed for the first test of all of them irrespective of the pattern type when the plate was heated up. It is believed that the most important effect for the uncertainty quantifications performed herein is that the speckle pattern may be altered between tests of the same series. Before each experiment was started, 30 pairs of pictures were acquired for uncertainty quantifications that are analyzed in the sequel. In that part of the test, no smoke was observed. However, from one test to the next, the speckle pattern had evolved. 
Table 2. Main features of the 14 laser impacts performed on 5 different plates and patterns (Figure 6). The observations were conducted with the pictures acquired by the logarithmic camera

\begin{tabular}{|c|c|c|c|c|}
\hline $\begin{array}{l}\text { Test } \\
\text { ID }\end{array}$ & $\begin{array}{c}\text { Illumination } \\
\text { duration }\end{array}$ & $\begin{array}{c}\text { Maximum } \\
\text { temperature }\end{array}$ & Dwell & Observations \\
\hline $1 \mathrm{a}$ & $10 \mathrm{~s}$ & $1400^{\circ} \mathrm{C}$ & $65 \mathrm{~min}$ & smoke \\
\hline $1 b$ & $3 \mathrm{~s}$ & $810^{\circ} \mathrm{C}$ & $45 \mathrm{~min}$ & no smoke \\
\hline $1 \mathrm{c}$ & $3 \mathrm{~s}$ & $810^{\circ} \mathrm{C}$ & - & no smoke \\
\hline $2 \mathrm{a}$ & $3 \mathrm{~s}$ & $1080^{\circ} \mathrm{C}$ & 36 min & $\begin{array}{l}\text { smoke }+ \\
\text { pattern degradation }\end{array}$ \\
\hline $2 b$ & $3 \mathrm{~s}$ & $910^{\circ} \mathrm{C}$ & $24 \mathrm{~min}$ & no smoke \\
\hline $2 \mathrm{c}$ & $3 \mathrm{~s}$ & $<600^{\circ} \mathrm{C}$ & 17 min & $\begin{array}{c}\text { no smoke, pyrometer } \\
\text { pointing changed }\end{array}$ \\
\hline $2 \mathrm{~d}$ & $3 \mathrm{~s}$ & $1120^{\circ} \mathrm{C}$ & - & no smoke \\
\hline $3 \mathrm{a}$ & $2 \mathrm{~s}$ & $680^{\circ} \mathrm{C}$ & $15 \mathrm{~min}$ & smoke \\
\hline $3 b$ & $2 \mathrm{~s}$ & $700^{\circ} \mathrm{C}$ & $17 \mathrm{~min}$ & no smoke \\
\hline $3 \mathrm{c}$ & $2 \mathrm{~s}$ & $710^{\circ} \mathrm{C}$ & $21 \mathrm{~min}$ & no smoke \\
\hline $4 \mathrm{a}$ & $2 \mathrm{~s}$ & $710^{\circ} \mathrm{C}$ & $14 \mathrm{~min}$ & smoke \\
\hline $4 b$ & $2 \mathrm{~s}$ & $720^{\circ} \mathrm{C}$ & $19 \mathrm{~min}$ & smoke (a bit) \\
\hline $5 \mathrm{a}$ & $2 \mathrm{~s}$ & $710^{\circ} \mathrm{C}$ & $19 \mathrm{~min}$ & smoke \\
\hline $5 \mathrm{~b}$ & $2 \mathrm{~s}$ & $740^{\circ} \mathrm{C}$ & - & smoke (a bit) \\
\hline
\end{tabular}

\#Indicative levels measured by a bichromatic pyrometer monitoring the center of the laser spot (apart from test 2c) 


\subsection{Correlation parameters}

All calibrations, shape corrections and displacement measurements performed herein were conducted within the EikoTwin DIC [44] framework. Table 3 gathers all analysis parameters.

Table 3. DIC analysis parameters

\begin{tabular}{|l|l|}
\hline \hline DIC software & EikoTwin DIC $[44]$ \\
Image filtering & rgb2gray \\
Element size & $1.12 \mathrm{~mm}$ \\
Shape functions & Linear (T3 elements) \\
Evaluation points (per element) & $55\left(i . e ., N_{e} \approx 7.4\right)$ \\
Matching criterion & Normalized quadratic differences \\
Interpolant & Linear \\
Displacement noise-floor & see text and Figure 16 \\
\hline \hline
\end{tabular}

The finite element mesh of the nominal $100 \times 100 \mathrm{~mm}^{2}$ plate surface is shown in Figure 10(a). A regular discretization was utilized with an element size of $1.12 \mathrm{~mm}$. Parts of the support overlapping with the plate surface were removed in addition to the central part of the plate that was illuminated by the laser. In that last area, the speckle pattern was altered by the laser source and the cameras were sensitive to near IR radiations even though blue filters were utilized. 


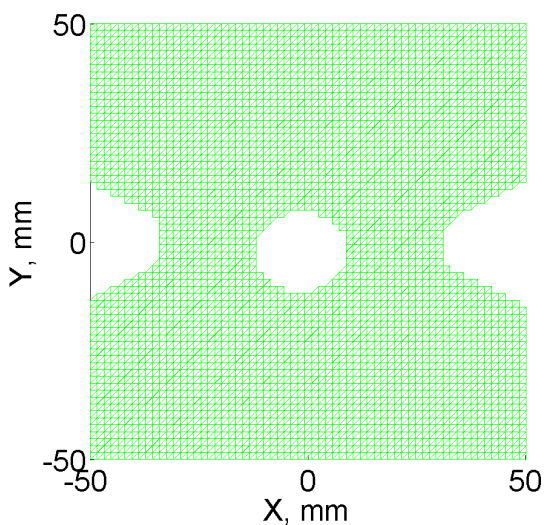

(a)
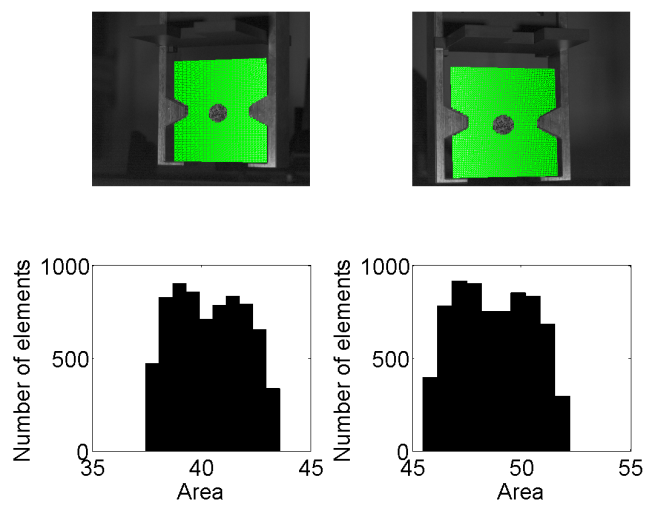

(b)

Figure 10. (a) Mesh of the nominal configuration. (b) Projected meshes onto the two pictures of the reference configuration of the first test, and corresponding histogram of surface areas of each element expressed in pixels

Figure 10(b) shows the meshes when reprojected onto the reference images acquired by both cameras before the very first test. Even though the initial mesh was structured, the meshes in both images were no longer uniform (see histograms of Figure 10(b)). The mean element size was equal to $6.4 \mathrm{px}$ for the first camera, and $7.0 \mathrm{px}$ for the second camera. The corresponding spatial resolution (i.e., the square root of the total number of pixels covered by the shape functions shared by each node) was equal to $15.2 \mathrm{px}$ and 16.7 px, respectively. Had all nodes had a connectivity of 6 (as inner nodes did), the spatial resolution would have been equal to $6.4 \sqrt{6}=15.7 \mathrm{px}$ and $6.4 \sqrt{6}=17.1 \mathrm{px}$, respectively.

Before performing displacement measurements, shape corrections with respect to the nominal model of the plate were performed [38]. This correction was carried out prior to each test since the temperatures were high enough to have residual deformations at the end of each laser impact. They were also performed when the plate was in its as received state since the plate thickness was not high (i.e., $2 \mathrm{~mm}$ ) and there were some flatness defects. Figure 11 shows the shape correction that had to be performed for test $2 \mathrm{a}$. 


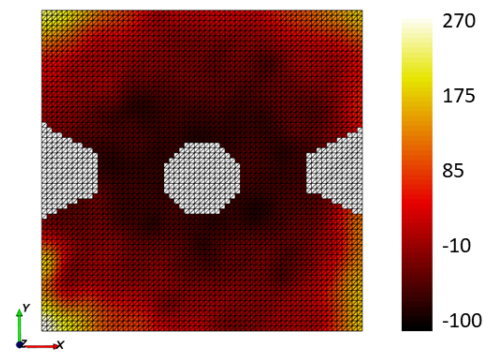

Figure 11. Shape correction (expressed in $\mu \mathrm{m}$ ) in the out-of-plane direction for test 2a

For illustration purposes, Figure 12 shows the out-of-plane displacement fields for three particular instants of the experiment, namely, when maximum "swelling" was observed, then maximum sinking, and at the end of the test. The maximum amplitudes were about $\pm 450 \mu \mathrm{m}$. These levels are more than one order of magnitude larger than the measurement uncertainties that will be discussed in the following sections.

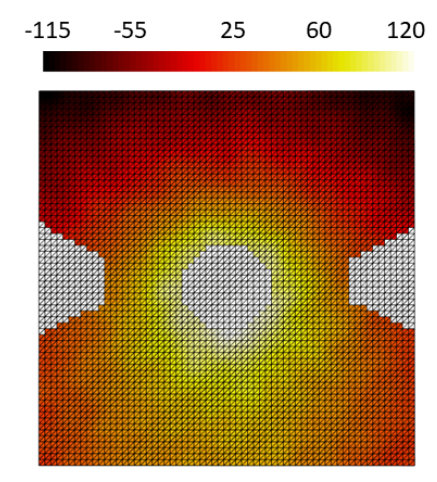

(a)

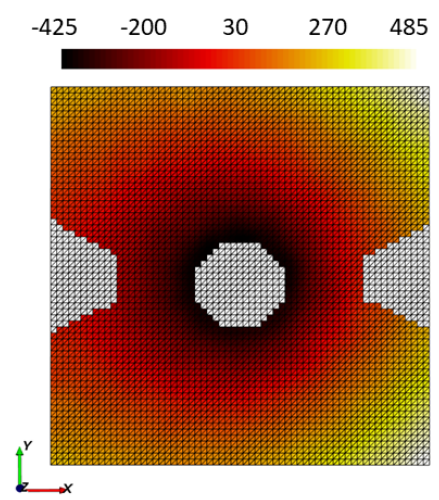

(b)

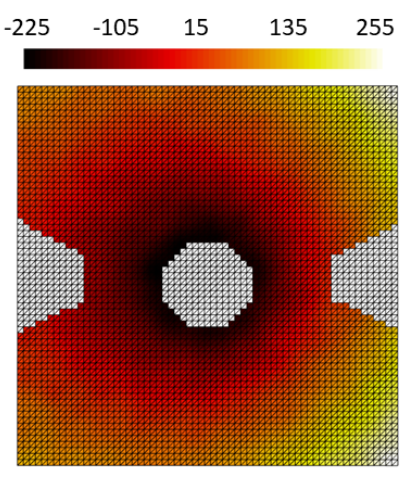

(c)

Figure 12. Out-of-plane displacement fields (expressed in $\mu \mathrm{m}$ ) for (a) maximum swelling, (b) maximum sinking, and (c) at the end of the test

The previous fields are available for any analyzed picture pair. In the following, five locations of virtual displacement sensors were selected, namely, one point close to the illuminated zone, and four points close to the corners of the plate (Figure 13(a)). 


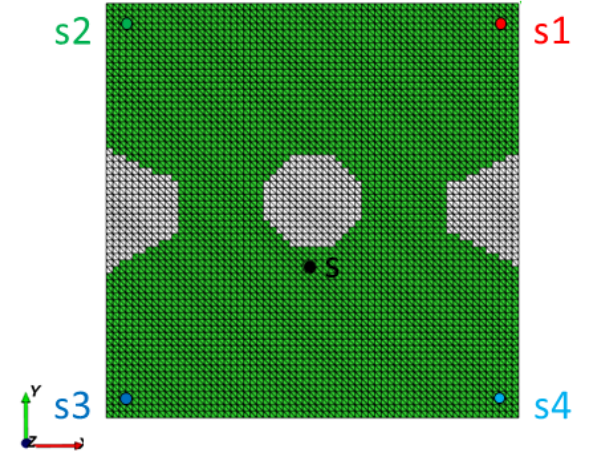

(a)

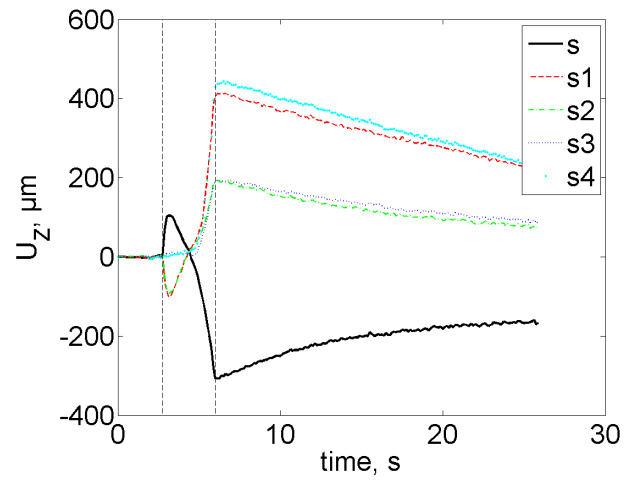

(b)

Figure 13. (a) Location of the five virtual sensors for which the out-of-plane motion $U_{Z}$ histories are reported in sub-figure (b). The vertical dashed lines depict the beginning and the end of laser illumination for test $2 \mathrm{a}$

Figure 13(b) shows the temporal displacement histories of these 5 points. Sensor $s$ has a temporal history that is out of phase with the other four because of the localized temperature gradient in the central part of the sample. Further, the sensors monitoring to the same side (i.e., $s 1-s 4$ and $s 2-s 3$ ) have very close responses (when $t>4.4 \mathrm{~s}$ ), yet different from one side to the other. This observation shows that the way the plate deformed during the experiment was not symmetric, which is also observed in Figure 12(b-c). At the beginning of the experiment, sensors $s 1$ and $s 2$ had similar histories, and sensors $s 3$ and $s 4$ as well. The inversion occurred when $t \approx 4.4 \mathrm{~s}$ for which the displacements were identical (and very small) for the five sensors. This global inversion is also observed when comparing the displacement fields reported in Figure 12(a) and Figures 12(b-c).

These last results are only a small part of the analysis of the experiments reported herein. As smoke was observed at least for the first test of each series (Table 2), it calls for spatiotemporal analyses in which spurious fluctuations can be mitigated thanks to temporal regularization $[45,46]$. However, special care has to be exercised to avoid filtering out meaningful parts of the signal. Another route may be so-called PGD- 
DIC $[47,48]$ in which spatiotemporal modes are constructed on the fly, some of them possibly having the signature of spurious motions induced by smoke. The remainder of the paper focuses on uncertainty quantifications in which both afore-mentioned enhancements were not considered.

\subsection{A Priori Estimates}

The mesh used in the present study was very fine (i.e., with an element size equal to $1.12 \mathrm{~mm}$, see Figure 10(a)). Consequently, when the number of integration points increases, one pixel may be shared by more than one integration point. In that case, no gain is expected in terms of measurement uncertainty since no additional information is available (i.e., the standard displacement uncertainties no longer decrease). It follows that an equivalent number of integration points is defined

$$
N_{e}=\min \left(N_{I P}, N_{p x}\right)
$$

where $N_{p x}$ is the maximum of the mean element size in the meshes reprojected in the camera planes (i.e., $7 \mathrm{px}$ in the present case). Equation (9) is modified to account for this limitation

$$
\sigma_{k}^{2}=\frac{\Sigma_{u}^{2}}{N_{e}^{2}} \sum_{c} \frac{4\left(\sigma^{c}\right)^{2}}{\left(\Pi_{k}^{c}\right)^{2}\left\langle\left\|\nabla I_{0}^{c}\right\|_{2}^{2}\right\rangle}
$$

where the number of integration points $N_{I P}$ is replaced by the equivalent number of integration points.

To validate the previous extension, a first test case consisted in considering the image pairs of the reference configuration of the first test, and artificially adding Gaussian white noise to each pixel with a standard deviation of 1.2 gray level (i.e., $\sqrt{2} \sigma^{c}=0.8$ gray level [25]) to create a series of 29 pairs to be representative of the experimental dataset available for uncertainty quantifications. Stereocorrelation analyses were conducted for different numbers of integration points on these image series. For each degree of freedom, the variance of its temporal fluctuations was computed. Then, for each direction, the square root of the mean level was finally calculated and compared to the a priori estimate provided by Equation (11) in Figure 14. 


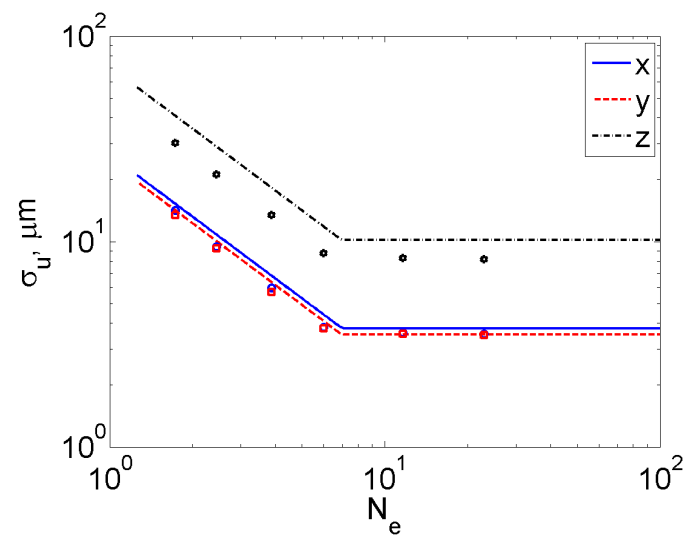

Figure 14. Standard displacement uncertainties $\sigma_{u}$ vs. equivalent number of integration points for the artificial test case. Curves: a priori estimates (Equation (11)), symbols: a posteriori evaluations

For the in-plane components, a very good agreement is observed between the a priori estimate and the correlation results. In particular, the flattening of the displacement uncertainties is very well captured. The standard uncertainty for the out-of-plane component over-estimates a bit the actual results. This may be due to the fact that the total sensitivities in the out-of-plane direction are less uniform than those in the in-plane directions (Figure 4).

The first set of results validates Equation (11) when applied to an artificial test case (i.e., when random errors with a priori no source of systemic error). They are in line with what was already observed in other configurations [25]. The next step will be to investigate the 14 tests in which additional sources of errors may be present (e.g., illumination changes, pattern degradation).

\subsection{A Posteriori Validations}

The same type of study as before was conducted on the 30 image pairs acquired prior to the first test. This series is representative of the true experimental conditions before the laser was turned on. One additional step was to determine the actual level of acquisition 
noise for both cameras. Since it has to be assessed for both cameras, 2D DIC analyses were run on each image series, and the gray level residuals were stored. For each pixel of the ROI, the standard deviation of the residuals was computed, and the mean over the ROI was evaluated. A 2/3 correction was finally applied since a bilinear gray level interpolation scheme was considered (i.e., $\sqrt{2} \sigma^{1}=1.2$ gray level, $\sqrt{2} \sigma^{2}=1.3$ gray level). This level was about $50 \%$ higher than in the artificial test case.

Figure 15 reports the change of the standard displacement uncertainties as functions of the equivalent number of integration points for the first test. Overall, a good agreement is observed, and a very good agreement for the plateau levels. This analysis shows that there is no need to use more than about 49 integration points in each element since there is no gain in terms of measurement uncertainty. Consequently, the first number compatible with the triangular geometry of the elements is $N_{I P}=10 \times 11 / 2=$ 55. It was used in all the following analyses (Table 3).

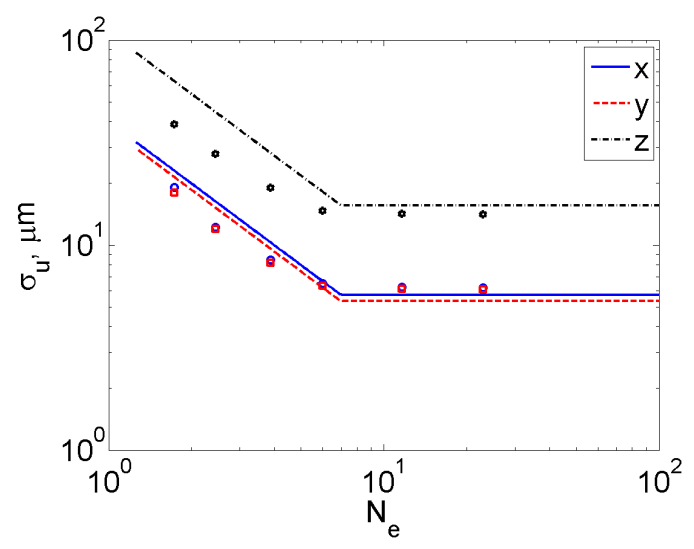

Figure 15. Standard displacement uncertainties $\sigma_{u}$ vs. equivalent number of integration points for the first test (1a). Curves: a priori estimates (Equation (11)), symbols: a posteriori evaluations

For the fourteen tests, stereocorrelation measurements were performed for the series of 30 image pairs. Each time, the reference pictures were those of the considered test even though only five different patterns were available, yet they may have degraded because of laser illumination (Table 2). Figure 16 shows the results for all uncertainty quantifications with the comparisons with the a priori estimates. A very good agreement is observed. Further, even though some pattern degradation was observed and the dwell 
duration was rather short for test series 3 to 5 , it did not influence the measurement uncertainties when repeated tests were analyzed (Table 2). 


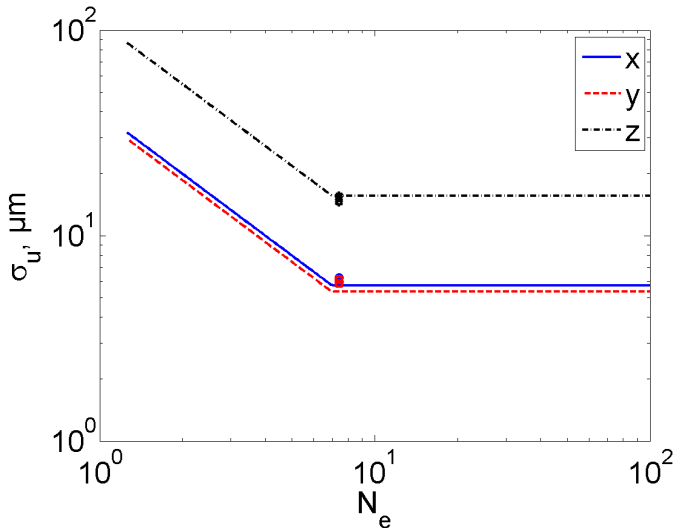

Pattern \#1

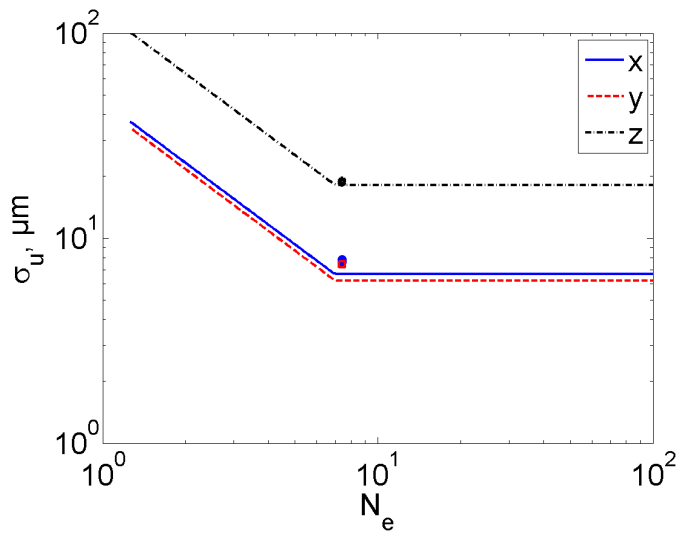

Pattern \#3

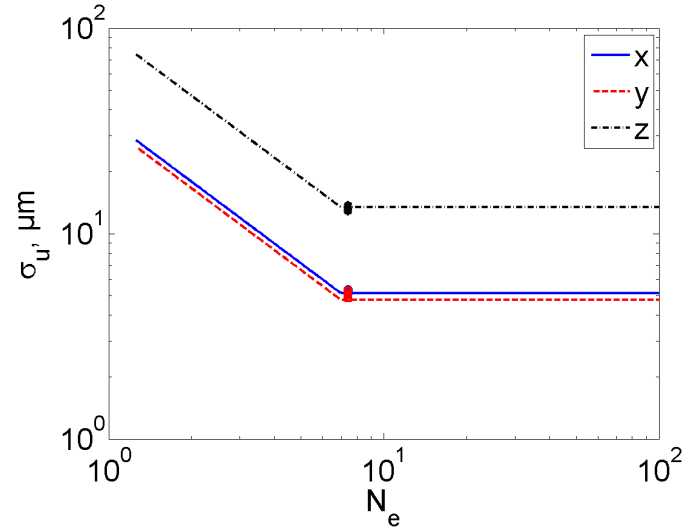

Pattern \#2

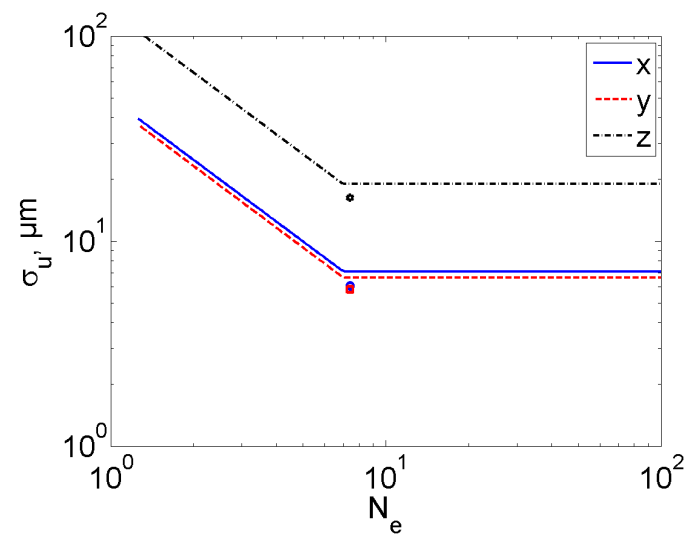

Pattern \#4

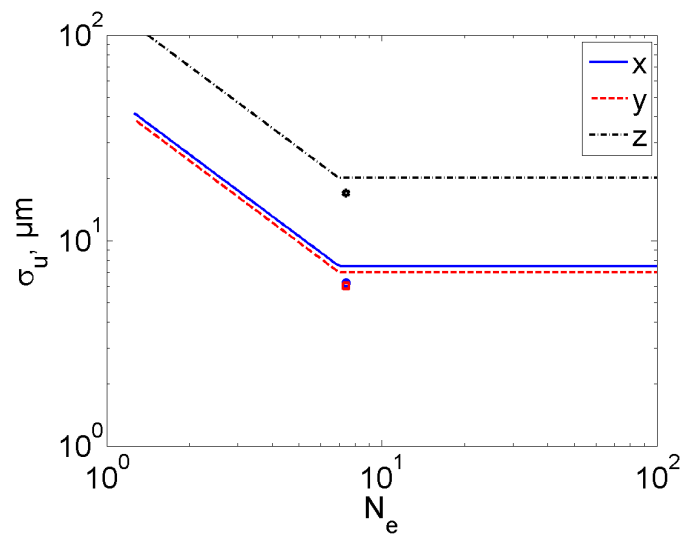

Pattern \#5

Figure 16. Standard displacement uncertainties $\sigma_{u}$ vs. equivalent number of integration points for all the tests. Curves: a priori estimates (Equation (11)), symbols: a posteriori evaluations

In Figure 17, the a priori estimates are compared to the a posteriori evaluations. 
As expected from Figure 16, a very good correlation (i.e., Pearson correlation coefficient $r=0.98)$ is observed, which validates the a priori estimates proposed herein. When a linear regression is performed, a slope of 0.96 is found between a priori and a posteriori displacement uncertainties.

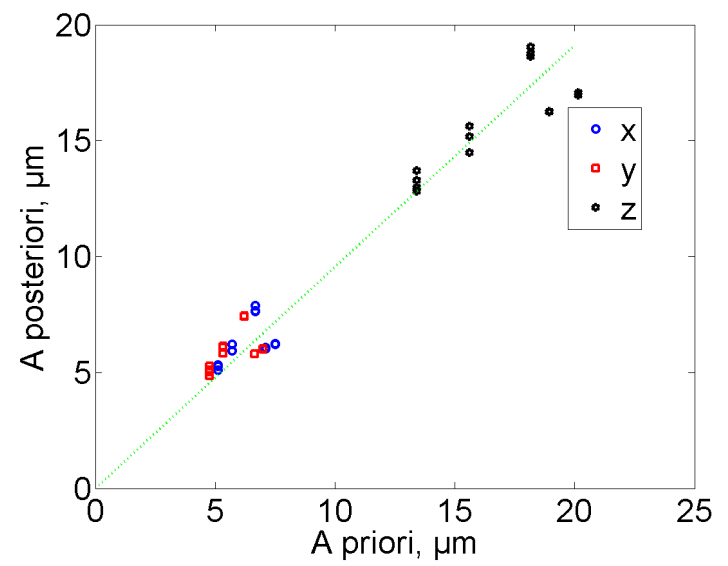

Figure 17. A posteriori standard displacement uncertainties $\sigma_{u}$ vs. a priori estimates (Equation (11)). The solid linear depicts the best linear fit (slope: 0.96, correlation coefficient: 0.98)

\subsection{Influence of Speckle Pattern}

Even though two different patterning techniques were investigated, no clear difference was observed in terms of measurement uncertainties (Figure 17). Further, the fact that each pattern was heated did not induce significant changes in the uncertainty levels, even though smoke was observed and the patterns were not all stable against laser illumination (Table 2). This effect was mitigated by excluding the zone that was impacted by the laser (Figure 10). All these observations show that measurement uncertainties are the result of an interplay between image contrast (here characterized by the RMS image gradient), the optical setup that determines the placement sensitivities (which depend on the extrinsic and intrinsic parameters of each camera), and acquisition noise (i.e., camera hardware and illumination). Consequently, the speckle pattern is only one of these numerous components, and in the present case, it had not a significant 
influence as the RMS image gradients were all of the order of 10 gray level per pixel (Figure 9).

\section{Conclusion}

A priori estimates of standard displacement uncertainties were considered when derived from mean field approximations of variances in an FE-based stereocorrelation framework. They were extended to account for their flattening when the distance between integration points becomes less than the pixel size. These expressions combine a measure of image contrast, placement sensitivities, number of integration points and acquisition noise.

Uncertainty quantifications of 14 thermomechanical tests were conducted on stainless plates that were laser-shocked (some for peak temperatures greater than $1000^{\circ} \mathrm{C}$ ). Five different patterns were considered, three of them nanostructured, and two speckled with B/W paints. Even though some repeated laser impacts were applied onto the same patterns, which experienced high temperatures, no degradation of the noise-floor displacements was observed.

For the selected experimental setup, the a priori estimates of standard displacement uncertainties were experimentally validated for all 14 tests. This result is, for example, very useful for designing and optimizing multiview systems (i.e., lowering the measurement uncertainties by suited camera placements [39]). Such uncertainty quantifications will have to be investigated for more complex shapes and surface geometries for which the mean field approximations utilized herein may be further challenged. 


\section{Acknowledgments}

This work was funded thanks to an Innovation Lab grant of MBDA France. It was performed under the framework agreement no. 10078 between ALPhANOV and MBDA. The logarithmic camera was kindly provided by NIT. The nanostructured speckle patterns were prepared by S. Nourry and G. Mincuzzi at ALPhANOV.

\section{References}

[1] ISO 1995 Guide to the Expression of Uncertainty in Measurements (GUM) (Geneva (Switzerland): International Organization for Standardization)

[2] ISO 1993 International Vocabulary of Basic and General Terms in Metrology (VIM) (Geneva (Switzerland): International Organization for Standardization)

[3] Sutton M, Orteu J and Schreier H 2009 Image correlation for shape, motion and deformation measurements: Basic Concepts, Theory and Applications (New York, NY (USA): Springer)

[4] Orteu J 2009 Optics and Lasers in Engineering 47 282-291

[5] Sutton M 2013 Applied Mechanics Reviews 65050802

[6] Luo P, Chao Y, Sutton M and Peters W 1993 Experimental Mechanics 33 123-132

[7] Luo P, Chao Y and Sutton M 1994 Optical Engineering 33 981-990

[8] Helm J, McNeill S and Sutton M 1996 Optical Engineering 35 1911-1920

[9] Orteu J J, Garric V and Devy M 1997 Camera calibration for 3D reconstruction: application to the measurement of 3D deformations on sheet metal parts New Image Processing Techniques and Applications: Algorithms, Methods, and Components II vol 3101 ed Refregier P and Ahlers R J International Society for Optics and Photonics (SPIE) pp 252-263

[10] F Hild and S Roux 2012 Method of three-dimensional stereo-correlation measurements using a parametric representation of the measurement object Patent family FR2991448B1, EP2856424B1, US9733071B2, ES2700975T3 URL patents.google.com/patent/FR2991448B1/en

[11] Dufour J E, Beaubier B, Hild F and Roux S 2015 Experimental Mechanics 55 1657-1668

[12] Dufour J E, Hild F and Roux S 2015 Journal of Strain Analysis for Engineering Design 50 470-487

[13] Dufour J, Beaubier B, Roux S and Hild F 2014 Displacement measurement using CAD-based stereo-correlation with meshes ICEM conference 
[14] Dubreuil L, Dufour J E, Quinsat Y and Hild F 2016 Experimental Mechanics 56 1231-1242

[15] Pierré J E, Passieux J C and Périé J N 2017 Experimental Mechanics 57 443-456

[16] Wang Y Q, Sutton M A, Ke X D, Schreier H W, Reu P L and Miller T J 2011 Experimental Mechanics 51 405-422

[17] Reu P 2013 Experimental Mechanics 53 1661-1680

[18] Balcaen R, Wittevrongel L, Reu P L, Lava P and Debruyne D 2017 Experimental Mechanics 57 $703-718$

[19] Zhu C, Yu S, Liu C, Jiang P, Shao X and He X 2019 Measurement Science and Technology 30 025204

[20] Ke X D, Schreier H W, Sutton M A and Wang Y Q 2011 Experimental Mechanics 51 423-441

[21] Berny M, Archer T, Beauchêne P, Mavel A and Hild F 2020 Submitted for publication

[22] Balcaen R, Reu P, Lava P and Debruyne D 2017 Experimental Mechanics 57 939-951

[23] Standardization, Good Practices, and Uncertainty Qunatification Committee A Good Practices Guide for Digital Image Correlation International Digital Image Correlation Society (iDICs)

[24] ASD-STAN prEN 4861 P1 2018 Metrological assessment procedure for kinematic fields measured by digital image correlation URL /www. asd-stan.org/downloads/asd-stan-pren-4861-p1

[25] Hild F and Roux S 2020 Journal of Strain Analysis for Engineering Design DOI: $10.1177 / 0309324720927102$

[26] Rao C 1945 Bulletin of the Calcutta Mathematical Society 37 81-89

[27] Cramér H 1946 Mathematical Methods of Statistics (Princeton, NJ (USA): Princeton Univ. Press)

[28] Faugeras O and Toscani G 1987 Camera calibration for 3D computer Vision International Workshop on Machine Vision and Machine Intelligence pp 240-247

[29] Faugeras O 1993 Three-dimensional computer vision: a geometric viewpoint (Cambridge, MA (USA): MIT Press)

[30] Anscombe F 1948 Biometrika 35 246-254

[31] Sur F and Grédiac M 2014 IEEE Signal Processing Letters 21 432-436

[32] Curt J, Capaldo M, Hild F and Roux S 2020 Optics and Lasers in Engineering 127105896

[33] Lyons J, Liu J and Sutton M 1996 Experimental Mechanics 36 64-70

[34] Novak M and Zok F 2011 Review of Sicentific Instruments 82115101

[35] Leplay P, Lafforgue O and Hild F 2015 Journal of the American Ceramic Society 98 2240-2247

[36] Jones E and Reu P 2018 Experimental Mechanics 58 1133-1156

[37] New Imaging Technologies NIT, Verrières le Buisson (France) 2020 MAGIC Camera (MC1003- 
1VF) URL http://new-imaging-technologies.com/cmos-products/

[38] Beaubier B, Dufour J, Hild F, Roux S, Lavernhe-Taillard S and Lavernhe-Taillard K 2014 Experimental Mechanics 54 329-341

[39] Vitse M, Poncelet M, Iskef A, Dufour J E, Gras R, Bouterf A, Raka B, Giry C, Gatuingt F, Hild F, Ragueneau F and Roux S 2020 Journal of Strain Analysis for Engineering Design DOI: $10.1177 / 0309324720910887$

[40] Vorobyev A and Guo C 2011 Applied Surface Science 257 7291-7294

[41] Tsibidis G, Fotakis C and Stratakis E 2015 Physical Review B 92(4) 041405

[42] Mincuzzi G, Rebière A, Goaec B L, Nourry S, Pagano P, Faucon M and Kling R 2019 Beam engineering for high throughput material processing with high power, femtosecond lasers Laserbased Micro- and Nanoprocessing XIII vol 10906 ed Klotzbach U, Watanabe A and Kling R International Society for Optics and Photonics (SPIE) pp 201-208

[43] Triconnet K, Derrien K, Hild F and Baptiste D 2009 Optics and Lasers in Engineering 47 728-737

[44] Eikosim 2020 (accessed) EikoTwin DIC URL eikosim.com/en/eikotwin-2/

[45] Besnard G, Leclerc H, Roux S and Hild F 2012 Journal of Strain Analysis for Engineering Design $47214-228$

[46] Berny M, Archer T, Mavel A, Beauchêne P, Roux S and Hild F 2018 Optics and Lasers in Engineering 40 544-556

[47] Berny M, Jailin C, Bouterf A, Hild F and Roux S 2018 Measurement Science and Technology 29 125008

[48] Passieux J C, Bouclier R and Périé J N 2018 Experimental Mechanics 58 1195-1206 